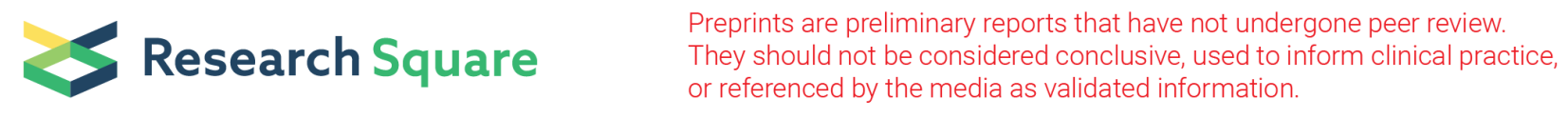

\title{
Two-Stage Total Knee Arthroplasty in the Treatment of Advanced Knee Septic Arthritis: Case Series
}

\author{
Hong Wang \\ Lanzhou University Second Hospital \\ Yuanjun Teng \\ Lanzhou University Second Hospital \\ Xingwen Wang \\ Lanzhou University Second Hospital \\ Shifeng Zhang \\ Lanzhou University Second Hospital \\ Tianen $\mathrm{Xu}$ \\ Lanzhou University Second Hospital \\ Hua HAN \\ Lanzhou University Second Hospital \\ Yayi Xia ( $\nabla$ xiayayilzu@sina.com) \\ Lanzhou University Second Hospital
}

Technical advance

Keywords: septic arthritis, knee infection, two-stage total knee arthroplasty, spacer

Posted Date: January 27th, 2021

DOI: https://doi.org/10.21203/rs.3.rs-154377/v1

License: (c) (i) This work is licensed under a Creative Commons Attribution 4.0 International License. Read Full License 


\section{Abstract}

PURPOSE: Septic arthritis (SA) is defined as the joint inflammation secondary to bacterial infection with a potentially fatal condition, but with no established approach treatment currently. Two-stage primary total knee arthroplasty (TKA) with an antibiotic-laden cement spacer has been reported to apply to treat SA of knee effectively in individual cases. We reported our cases aimed to contribute to current information on two-stage TKA in the treatment of advanced SA in knee joint.

Methods: We retrospectively screened clinical profiles of eleven patients with SA in knee joint who had two-stage TKA with an antibiotic-laden cement spacer. Information of inflammatory serological markers were summarized and statistic comparisons were done on Hospital for Special Surgery (HSS) score as well as the degree of the range of motion (ROM) between pre-, interval- and post-operation, with significant level of $p<0.05$.

Results: Infection in knee joint was eliminated in all eleven patients by two-stage TKA with an antibiotic-laden cement spacer. CRP took $3.4 \pm 1.4$ weeks (range, 1-6 weeks) to return to normal level, whilst for ESR was $16.3 \pm 3.0$ weeks (range, 13-23 weeks). Means of HSS score and ROM degree were significantly increased after both the first and second-stage surgery $(p<0.05)$. HSS score of three timings were not different in age, gender, micro-organism culture results and comorbidities in our study.

Conclusions: Two-stage TKA is an effective treatment in SA in knee joint in controlling infection, relieving clinical severity and improving function of keen joint thereafter.

Level of evidence IV.

\section{Introduction}

Septic arthritis (SA) is an emergency condition of joint infection and $40-50 \%$ of the adult patients were affected in the knee ${ }^{1-3}$. Patients of SA were always misdiagnosed of rheumatic disease and resulted to inadequate treatment ${ }^{4}$, which leads to irreversible joint destruction and even case-fatality (i.e., $\left.3-29 \%\right)^{5}$. Therefore, it is indeed important to diagnose this condition early and give effective treatment immediately ${ }^{1}$. The standard diagnosis of SA is based on detection of bacteria in synovial fluid by joint puncture. Primary therapy of SA includes the surgical intervention such as arthroscopic or open irrigation and debridement, concerted with effective antibiotics utilization. Arthroscopic surgery has been reported to play effective roles in SA treatment ${ }^{6}$, whilst open irrigation and debridement is usually considered for the patients of chronic infectious arthritis or persistent infection after arthroscopic surgery $4,7,8$. However, there are still cases that could not recover satisfactorily after such primary treatment, and eventually developed into non-salvageable destructive arthritis ${ }^{3}$.

Some prospective pilot studies were proposed and suggested that implementing interventional articulate cement spacer in the problematic joint then perform the TKA resulted more effectiveness in control of infection and improvement of functional outcomes ${ }^{3,9-11 . ~}$

Previous studies were mostly case reports ${ }^{3,11-13}$, and evidences regarding the therapy aspect was limited. Therefore, this study aims to contribute to existing literature and provide insights into this important topic for future research.

\section{Methods \\ Participants}

We retrospectively collected and investigated eleven eligible patients attending the Department of Orthopedic of Second Hospital of Lanzhou University in China from January 2014 to December 2020. This protocol was approved by the Medical Ethical Committee of the Second Hospital of Lanzhou University (Supplement), and informed written consent was obtained from participant/guardian. All the patients were diagnosed of SA ${ }^{14,15}$ and treated by a two-stage arthroplasty for advanced knee SA consecutively (Fig. 1). All the surgeries were conducted by one surgical team. Deep infection was documented in all eleven cases at the time of initial debridement. We applied no age or gender limitation. All patients had their follow-up at our institution.

\section{Surgical technique and perioperative care}

\section{First stage}

In the first stage, a conventional medline knee skin incision is performed, followed by exposure of the joint via the parapatellar medial approach and complete removal of the synovial membrane, as well as infected and necrotic tissue. An intra-operative frozen section and bacterial cultivation of synovium and joint fluid were taken to confirm the diagnosis and guide the choice of susceptible antibiotic, three biopsies were taken during first-stage procedure for postoperative pathological examination (Fig. 2). Bone cutting was performed in preparation for primary TKA according to the size of the spacer, and all cuts were performed under extramedullary cutting guides to avoid intramedullary contamination. The measurement was performed in the full length image of lower limbs as shown in Fig. 3. Line from point A (i.e., the center of femoral condyle) to point $D$ (i.e., the center of femoral head) and the line from point B to ponint $C$ (i.e., connecting line of the anterior superior iliac spine on both sides) were drawn for the corss point $E$, and the distance between point $B$ to point $E$ was measured and marked. During the operation, line from point $A$ and point $E$ was the virtual mechanical alignment of the lower limb, and finally the cutting line of the distal femur was the vertical line of the line between point A and point E. A spacer mold (Stage One; LINK) was used to create a dynamic antibiotic spacer which was constructed using 80 gram of Palacos $R+G$ bone cement (Heraeus Medica, Hanau, Germany) mixed with gentamycin (4.8 gram) and vancomycin (8 gram). The cement was mixed without vacuum using a "poor mixing technique" to improve antibiotic elution ${ }^{16}$. The antibiotic-loaded bone cement was poured into the spacer block mold at liquid stage, which was shaped as the prosthesis and taken out at the curing period. The antibiotic spacer 
was then applied to the bone surfaces using a poor bonding technique, which allows some blood to get between the spacer and the bone and for some motion to occur in the knee prior to cement hardening, to facilitate later removal. The femoral spacer block was inserted first and then an appropriate tibial spacer block adjusted by the extension gap was inserted so that the limb was aligned and tissue tension was slight relax for better postoperative range of motion (Fig. 4). After numerous irrigations the soft tissues were closed with the use of a suction drain for less than 48 hours.

\section{Second stage}

Total knee arthroplasty was performed at least 12 weeks later when laboratory inflammation markers had normalized and there was no further clinical evidence of infection (reddened and hyperthermic joint, drainage from the wound, unreasonable knee pain), intraoperative selection cemented posterior stabilized prosthesis (Smith \& Nephew® Primary Total Knee System, GENESIS II) was implanted patella replacement was not performed (Fig. 5).

\section{Antibiotic treatment and perioperative care}

After the first-stage surgery, a two-week intravenous anti-biotherapy was administered according to bacterial culture, and sensitive results whereas vancomycin was used for those without culture and sensitive results. Oral antibiotics (rifampicin + [optimal antibiotics/levofloxacin]) were continued for six weeks until two successive C-reactive protein (CRP) results were normal and erythrocyte sedimentation rate (ESR) results were below twice the value of the high threshold or had a continuous downtrend with no positive signs and symptoms. The CRP and ESR were followed every three days in the first two weeks and every two weeks thereafter.

During the second stage surgery, we applied vancomycin 1 gram prophylaxis in the implantation of primary TKA surgical procedures ${ }^{17}$, and postoperatively vancomycin was continued until wounds were dry. Then treatment was changed to oral rifampicin and levofloxacin for another 24 weeks ${ }^{18}$. Professional rehabilitation and functional training were undertaken by rehabilitation physiatrists in our hospital after stages, and all patients were followed up every three months in the first year, and once per year after.

\section{Statistical analysis}

Patient's files were screened by two authors for residual pain post-operative complications (including hematoma, seroma, blood transfusion, deep venous thrombosis, and revision surgeries). The repeated measurement of Hospital for Special Surgery Knee (HSS) score among pre-operation (i.e., before the firststage TKA surgery), interval period (i.e., after first-stage and before the second stage TKA surgery), and post-operation (i.e., the latest follow-up) were compared using analysis of variance (ANOVA) and Post-hoc test of multiple group comparison. To determine the effect of a two-stage operation, the degree of pre-, interval- and post- knee range of motion (ROM) were compared using paired t-tests. Two-tailed independent t-test was applied to the comparisons between groups of age (i.e., before or after middle age, following PubMed age filtration of 65 years old), gender, micro-organism culture result (i.e., staphylococcus or negative) and comorbidities (i.e., have or not have). Data were reported in mean \pm standard deviation (SD). All statistical analyses were performed using SPSS software version 19.0 (SPSS Inc., Chicago, IL). The level of significance was set at $p<0.05$.

\section{Results}

Eleven patients of six males and five females were reported in this case-series (Table 1). Their mean age was $54.1 \pm 15.6$ years (range, 22-74 years). 81.8\% had invasive treatment such as knee injection (KI) (i.e., in 4/11), arthroscopic debridement (AB) (i.e., in 6/11) and open debridement (OB) (i.e., in 7/11) previously. Four patients had comorbidities might compromise immunity: one patient was diabetic, two patients were hypertensive and another had both. Regarding the micro-organism culture, six had positive results of staphylococcus and the other five were negative, though utilized antibiotics variously. 
Table 1

Demographics and history of patients

\begin{tabular}{|c|c|c|c|c|c|c|c|c|c|c|c|c|}
\hline Patient & $\begin{array}{l}\text { Gender } \\
\text { /Age }\end{array}$ & $\begin{array}{l}\text { Previous } \\
\text { operation }\end{array}$ & $\begin{array}{l}\text { Micro-organism } \\
\text { on culture }\end{array}$ & Comorbidity & $\begin{array}{l}\text { Antibiotics } \\
\text { (weeks) }\end{array}$ & $\begin{array}{l}\text { AORI } \\
\text { classification }\end{array}$ & $\begin{array}{l}\text { Interval } \\
\text { peirod } \\
\text { (weeks) }\end{array}$ & $\begin{array}{l}\text { CRP } \\
\text { recover } \\
\text { time } \\
\text { after } \\
\text { 1st - } \\
\text { stage } \\
\text { (week) }\end{array}$ & $\begin{array}{l}\text { ESR } \\
\text { recover } \\
\text { time } \\
\text { after } \\
\text { 1st- } \\
\text { stage } \\
\text { (week) }\end{array}$ & $\begin{array}{l}\text { Pre- } \\
\text { interval- } \\
\text { post } \\
\text { HSS }\end{array}$ & $\begin{array}{l}\text { Pre- } \\
\text { interval- } \\
\text { post } \\
\text { RMO } \\
\text { (degree) }\end{array}$ & $\begin{array}{l}1 \\
1 \\
1\end{array}$ \\
\hline 1 & $F / 51$ & $\mathrm{KI} / \mathrm{AB} / \mathrm{OB}$ & $\begin{array}{l}\text { Staphylococcus } \\
\text { aureus }\end{array}$ & None & 8 & Type 1 & 12 & 5 & 17 & $\begin{array}{l}26-63- \\
85\end{array}$ & $\begin{array}{l}30-70- \\
85\end{array}$ & 2 \\
\hline 2 & $\mathrm{~F} / 74$ & $\mathrm{KI} / \mathrm{OB}$ & Negative & DM, HTN & 6 & Type 1 & 12 & 2 & 19 & $\begin{array}{l}47-65- \\
87\end{array}$ & $\begin{array}{l}80-80- \\
100\end{array}$ & $<$ \\
\hline 3 & $M / 22$ & $A B / O B$ & $\begin{array}{l}\text { Staphylococcus } \\
\text { aureus }\end{array}$ & None & 24 & Type 2 & 13 & 1 & 13 & $\begin{array}{l}14-55- \\
78\end{array}$ & $\begin{array}{l}30-40- \\
60\end{array}$ & $\vdots$ \\
\hline 4 & $M / 52$ & $\mathrm{KI} / \mathrm{OB}$ & $\begin{array}{l}\text { Staphylococcus } \\
\text { aureus }\end{array}$ & None & 8 & Type 1 & 12 & 4 & 23 & $\begin{array}{l}13-59- \\
81\end{array}$ & $\begin{array}{l}30-70- \\
90\end{array}$ & $\vdots$ \\
\hline 5 & $F / 56$ & OB & $\begin{array}{l}\text { Staphylococcus } \\
\text { epidermidis }\end{array}$ & None & 7 & Type 1 & 12 & 3 & 17 & $\begin{array}{l}52-67- \\
87\end{array}$ & $\begin{array}{l}85-90- \\
100\end{array}$ & $\vdots$ \\
\hline 6 & $F / 59$ & $A B / O B$ & Negative & DM & 4 & Type 1 & 12 & 2 & 14 & $\begin{array}{l}34-59- \\
74\end{array}$ & $\begin{array}{l}40-55- \\
70\end{array}$ & ? \\
\hline 7 & $M / 68$ & None & $\begin{array}{l}\text { Staphylococcus } \\
\text { aureus }\end{array}$ & HTN & 6 & Type 2 & 14 & 3 & 15 & $\begin{array}{l}57-76- \\
92\end{array}$ & $\begin{array}{l}90-100- \\
100\end{array}$ & 1 \\
\hline 8 & $M / 64$ & $A B$ & Negative & None & 4 & Type 1 & 12 & 6 & 13 & $\begin{array}{l}44-76- \\
86\end{array}$ & $\begin{array}{l}90-80- \\
90\end{array}$ & 1 \\
\hline 9 & $\mathrm{M} / 31$ & $\mathrm{KI} / \mathrm{AB} / \mathrm{OB}$ & $\begin{array}{l}\text { Staphylococcus } \\
\text { aureus }\end{array}$ & None & 8 & Type 2 & 24 & 4 & 18 & $\begin{array}{l}29-81- \\
89\end{array}$ & $\begin{array}{l}30-90- \\
100\end{array}$ & 1 \\
\hline 10 & $F / 52$ & $A B$ & Negative & None & 8 & Type 1 & 12 & 3 & 15 & $\begin{array}{l}60-81- \\
91\end{array}$ & $\begin{array}{l}100- \\
100-110\end{array}$ & 1 \\
\hline 11 & $M / 66$ & None & Negative & HTN & 6 & Type 1 & 14 & 4 & 15 & $\begin{array}{l}47-79- \\
87\end{array}$ & $\begin{array}{l}50-90- \\
100\end{array}$ & 7 \\
\hline
\end{tabular}

Pre: assessed before the first stage surgery; interval: assessed before the second stage surgery; post: assessed at the latest follow-up. F: female; M: male; KI: injection; AB: arthroscopic debridement; OB: open debridement; ROM: range of motion; HSS: Hospital for Special Surgery Knee score; DM: diabetes; HTN: hypertension; CRP: C-reactive protein; ESR: erythrocyte sedimentation rate; AORI: Anderson Orthopaedic Research Institute.

Infection was controlled successfully in all patients by using this two-stage method for TKA. Time for CRP returned to normal level was $3.4 \pm 1.4$ weeks (range, 1-6 weeks), whilst for ESR was $16.3 \pm 3.0$ weeks (range, $13-23$ weeks). Duration of interval period were $14.4 \pm 4.2$ weeks (range, 10-24 weeks) (Table 1). The mean HSS score of pre-operation was $38.5 \pm 16.4$ (range, 13-60), in the interval period was $69.2 \pm 9.7$ (range, 55-81) and in the post-operation was $85.2 \pm 5.5$ (range 74-92) (Table 1, Fig. 6a). Comparisons of HSS score among these three timings were shown in the Fig. $6 \mathrm{a}$, which significantly increased $(p=0.000)$ after both the first $(p=0.000)$ and second-stage surgery $(p=0.000)$ (Fig. 6a). There was no significant difference identified of the HSS score in these three timings in age, gender, micro-organism culture result and comorbidities. The degree of ROM was obviously increased $(p=0.030)$ after both first $(p=0.012)$ and second-stage surgery ( $p=0.035$ ) (Fig. 6b), which was $54.5 \pm 31.8$ (range, 20-100) of pre-operation, $76.8 \pm 20.9$ (range 35-100) of interval period and 90.5 \pm 16.7, (range, 55-110) of post- operation (Table 1). Average time of follow-up was $14.4 \pm 4.2$ months (range, 10-45 months).

\section{Discussion}

The treatment principle of knee SA to date includes surgical treatment and appropriate antibiotics ${ }^{19}$. However, the effect of the above treatments on advanced infectious arthritis is very poor, yet there is no established guidelines for such conditions, especially in the demanding adults. Though limited therapeutic options, there were several researches put effects on applying two-stage approach to primary TKA and tried to improve the efficiency 2,3,11,20. In our study, demands in eliminating infection, relieving symptoms and returning function were fulfilled, Also, we reported similar experience and endings as previous studies, though with small sample size. Therefore, two-stage TKA could be treated as a potential option in sever knee SA management.

Patients enrolled to this study were all diagnosed of knee SA, which fulfilled the criteria of badly damaged knee joint, exfoliated cartilage and failure in controlling infection by arthroscopy and open debridement. We referred the operation mode from B Hochreiter et al. ${ }^{11}$. Regarding the femoral cutting, we applied the simple extramedullary guiding method in first stage, which achieved the therapeutic outcomes of decreasing risk of surgery-related infection spreading and better function recovery. Previous study reported ${ }^{21}$ that valgus angles in $30-51 \%$ of patients who received TKA were ranged beyond the Valgus Cut Angle (i.e., 5 to 7 degrees), which unfitted to intramedullary guide tools, and such unfittness resulted the malalignment of knee after TKA. Thus, in the firststage, we applied the simple extramedullary guiding method in cutting distal femur to all eleven patients, which was equivalent to individualized valgus, and the alignment of all eleven patients were approprate. In addition, extramedullary guiding method could reduce the spread of infection and decrease the risk of bleeding and fat embolism. Further more, this extramedullary guiding method does not need C-arm fluoroscopy for femoral head during operation which could reduce radiation injury of patient. 
CRP of all eleven patients were returned to normal level within six weeks after the first stage, though ESR decreasing slower. Previous studies suggested that spacer surface biofilm formation due to long interval period would casue infection recurrence. Our patients were suggested to receive the second-stage surgery within three months, whereas fulfill the criteria that $\geq 2$ times CRP test results in normal range, ESR halfly decreased and no infected symptoms such as joint redness, fever, wound drainage and unreasonable knee pain.

Infection were eliminated in all eleven patients, and their knee joint function also returned to the level for supporting daily activities. However, patient 3 and 6 did not fully follow our advice on functional exercise after the first stage, which resulted rigid knee joint surrounded by scar tissues. Thus, we had to perform the tibial tubercle osteotomy to expose the joint clearly and help to finish the operation in the second-stage surgery. Nevertheless, they had smaller ROM than other patients after surgery. We emphasized and strengthened functional exercise to them, including knee flexion and extension, lower limb muscle strength exercise and partial-weight bearing exercise, which accelerated their recovery in post-operation. In corresponding to our results, the knee joint function (i.e., HSS score) would significantly increase after both two stages. Therefore the function exercise should be insisted across the whole process especially within one month after each operation, which do affects the effect of this surgery.

Previous studies on age and gender mainly focused on patients with osteoarthritis undergoing primary TKA, which reported that patients received primary TKA of female were averagely postponeed three years later than male and had lower Knee Function scores than men preoperatively and postoperatively 22 . Such fingings demonstrated that earlier initiation of treatment may enhance postoperative outcomes in women. Another study ${ }^{23}$ revealed that female gender, younger age and worse preoperative pain predict greater risk of moderate-severe pain postoperatively in patients with primary and revision TKA. However, the information on such factors influcing the functional outcomes of two-stage TKA of knee SA is indeed scant. We did the comparisons on age, gender, microorganism culture result and comorbidities but failed to identify difference. Otherwise, previous studies had only focused on elderly patients and we performed two-stage TKA in two young patient (i.e., 22,31 years old) whose knee joint were with serious damaging, cartilage denudation and flexion deformity. Arthroscopic and open debridement failed to control his advanced infection. Eventually we had to perform two-stage TKA to save him from joint arthrodesis. However, further studies of randomized controlled trial and appreciate sample size are expected.

However, just like many previous studies $2,11,20,24$, our study has some limitations. First of all, our study was a retrospective study with a limited number of patients, the reason is that advanced knee arthritis combined with severe knee joint disability is not common but is difficult to treat. Second, we had no control group.Though small sample size, our study provided valuable information to this rare clinical challenge. We could conclude that the two-stage TKA with antibiotic-laden cement spacer should be considered as a potential option in the treatment of non-salvageable knee SA, as well as the application of extramedullary guiding method.

Conclusion: Two-stage TKA with extramedullary guiding method is an effective treatment in SA in knee joint in controlling infection, relieving clinical severity and improving function of keen joint thereafter.

\section{Abbreviations}

SA:septic arthritis; TKA:total knee arthroplasty; HSS: Hospital for Special Surgery; ROM:range of motion; CRP:C-reactive protein; ESR:erythrocyte sedimentation rate;

\section{Declarations}

\section{Authors' contributions}

Hong WANG, Yuanjun TENG, Xingwen WANG, Shifeng ZHANG, Tianen XU, Hua HAN and Yayi XIA were involved in the conceptual discussion and design of the review, in the critical appraisal of the content and have given final approval to the version to be published.

\section{Funding}

The National Natural Science Foundation of China (81874017, 81960403 and 82060413);

Lanzhou Science and Technology Plan Program (2018-3-52);

Cuiying Scientific and Technological Innovation Program of Lanzhou University Second Hospital (CY2017-QN12, CY2017-ZD02);

The Fundamental Research Funds for the Central Universities (Izujbky-2020-kb17);

TCM inheritance and Innovation Platform construction project of Lanzhou University Second Hospital (TCM-IPC-2020-05)

\section{Availability of data and materials}

The datasets used and/or analysed during the current study are available

from the corresponding author on reasonable request.

\section{Ethics approval and consent to participate}

The protocol for this study was approved by the Scientific Ethics Committee 
of the Second hospital of Lanzhou University.

According to a retrospective design of the study, informed consent was

waived and the authors had permission to access the data of patients in this

study.

\section{Consent for publication}

All data generated or analyzed during this study are included in this published article.

\section{Competing interests}

The authors declare that they have no competing interests.

\section{Contributor Information}

Hong Wang, Phone: 15193107527, Email: 414747058@qq.com.

Yuanjun Teng, Email: tengyj06@126.com.

Xingwen Wang, Email: 1069713678@qq.com.

Shifeng Zhang, Email: zsf199171@163.com.

Tianen Xu, Email: 1159166491@qq.com

Hua Han*,Email: 13993166655@126.com

Yayi Xia*,Email: xiayayilzu@sina.com

\section{References}

1. García-Arias M, Balsa A, Mola EM. Septic arthritis. Best Pract Res Clin Rheumatol 2011;25(3):407-421.

2. Nazarian DG, de Jesus D, McGuigan F, Booth RE, Jr. A two-stage approach to primary knee arthroplasty in the infected arthritic knee. J Arthroplasty. 2003;18(7 Suppl 1):16-21.

3. Kirpalani PA, In Y, Choi NY, Koh HS, Kim JM, Han CW. Two-stage total knee arthroplasty for non-salvageable septic arthritis in diabetes mellitus patients. Acta Orthop Belg. 2005;71(3):315-320.

4. Mathews CJ, Weston VC, Jones A, Field M, Coakley G. Bacterial septic arthritis in adults. Lancet . 2010;375(9717):846-855.

5. Balabaud L, Gaudias J, Boeri C, Jenny JY, Kehr P. Results of treatment of septic knee arthritis: a retrospective series of 40 cases. Knee Surg Sports Traumatol Arthrosc. 2007;15(4):387-392.

6. Vispo Seara JL, Barthel T, Schmitz H, Eulert J. Arthroscopic treatment of septic joints: prognostic factors. Arch Orthop Trauma Surg. 2002;122(4):204-211.

7. Stutz G, Kuster MS, Kleinstück F, Gächter A. Arthroscopic management of septic arthritis: stages of infection and results. Knee Surg Sports Traumatol Arthrosc : official journal of the ESSKA. 2000;8(5):270-274.

8. Wang C, Lee YH, Siebold R. Recommendations for the management of septic arthritis after ACL reconstruction. Knee Surg Sports Traumatol Arthrosc : official journal of the ESSKA. 2014;22(9):2136-2144.

9. Bauer T, Lacoste S, Lhotellier L, Mamoudy P, Lortat-Jacob A, Hardy P. Arthroplasty following a septic arthritis history: a 53 cases series. Orthop Traumatol Surg Res. 2010;96(8):840-843.

10. Bae DK, Yoon KH, Kim HS, Song SJ. Total knee arthroplasty in stiff knees after previous infection. The Journal of bone and joint surgery British volume. 2005;87(3):333-336.

11. Hochreiter B, Strahm C, Behrend H. Short-interval two-stage approach to primary total knee arthroplasty for acutely septic osteoarthritic knees. Knee Surg Sports Traumatol Arthrosc. 2016;24(10):3115-3121.

12. Moyad TF. Two-stage arthroplasty for the treatment of chronic osteomyelitis after routine arthroscopic knee surgery. Orthopedics. 2013;36(1):e109-112.

13. Supreeth S, Al Ghanami S, Shanmugasundaram S, Al Rawi RS, Abdawani AR, Abdelmasih SR. Successful two-stage primary total knee arthroplasty for infective arthritis of the knee - our experience. J Clin Orthop Trauma. 2020;11(Suppl 5):S746-s751.

14. Goldenberg DL, Cohen AS. Acute infectious arthritis. A review of patients with nongonococcal joint infections (with emphasis on therapy and prognosis). Am J Med. 1976;60(3):369-377.

15. Eder L, Zisman D, Rozenbaum M, Rosner I. Clinical features and aetiology of septic arthritis in northern Israel. Rheumatology (0xford). 2005;44(12):15591563.

16. Lewis G. Properties of antibiotic-loaded acrylic bone cements for use in cemented arthroplasties: a state-of-the-art review. J Biomed Mater Res B Appl Biomater. 2009;89(2):558-574. 
17. Anderson DJ, Podgorny K, Berríos-Torres SI, et al. Strategies to prevent surgical site infections in acute care hospitals: 2014 update. Infect Control Hosp Epidemiol. 2014;35 Suppl 2:S66-88.

18. Osmon DR, Berbari EF, Berendt AR, et al. Executive summary: diagnosis and management of prosthetic joint infection: clinical practice guidelines by the Infectious Diseases Society of America. Clin Infect Dis. 2013;56(1):1-10.

19. Molyneux EM, Lavy CB, Lavy VR, Walsh AL. Septic arthritis. Lancet. 1998;351(9108):1060-1061.

20. Shaikh AA, Ha CW, Park YG, Park YB. Two-stage approach to primary TKA in infected arthritic knees using intraoperatively molded articulating cement spacers. Clin Orthop Relat Res. 2014;472(7):2201-2207.

21. Bardakos N, Cil A, Thompson B, Stocks G. Mechanical axis cannot be restored in total knee arthroplasty with a fixed valgus resection angle: a radiographic study. J Arthroplasty. 2007;22(6 Suppl 2):85-89.

22. Parsley BS, Bertolusso R, Harrington M, Brekke A, Noble PC. Influence of gender on age of treatment with TKA and functional outcome. Clin Orthop Relat Res. 2010;468(7):1759-1764

23. Singh JA, Gabriel S, Lewallen D. The impact of gender, age, and preoperative pain severity on pain after TKA. Clin Orthop Relat Res. 2008;466(11):27172723.

24. Aseem Arif Shaikh MS, Chul-Won Ha MD. Two-stage Approach to Primary TKA in Infected Arthritic Knees Using Intraoperatively Molded Articulating Cement Spacers. Clin Orthop Relat Res. (2014);472:2201-2207

\section{Figures}
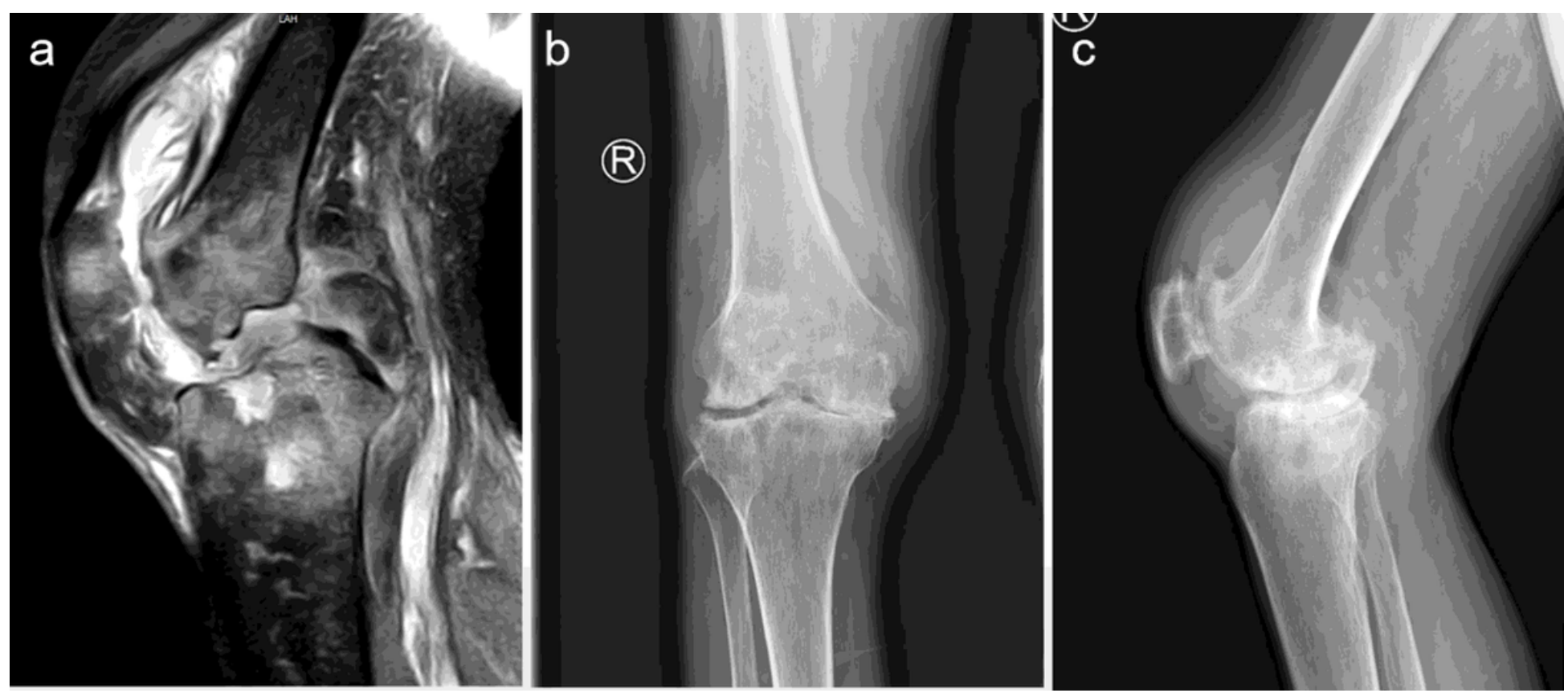

\section{Figure 1}

Images before surgery. (a) MRI PD-SPAIR image in Sagittal view; (b) X-ray image inanteroposterior (AP) view; (c) X-ray image in lateral view. 

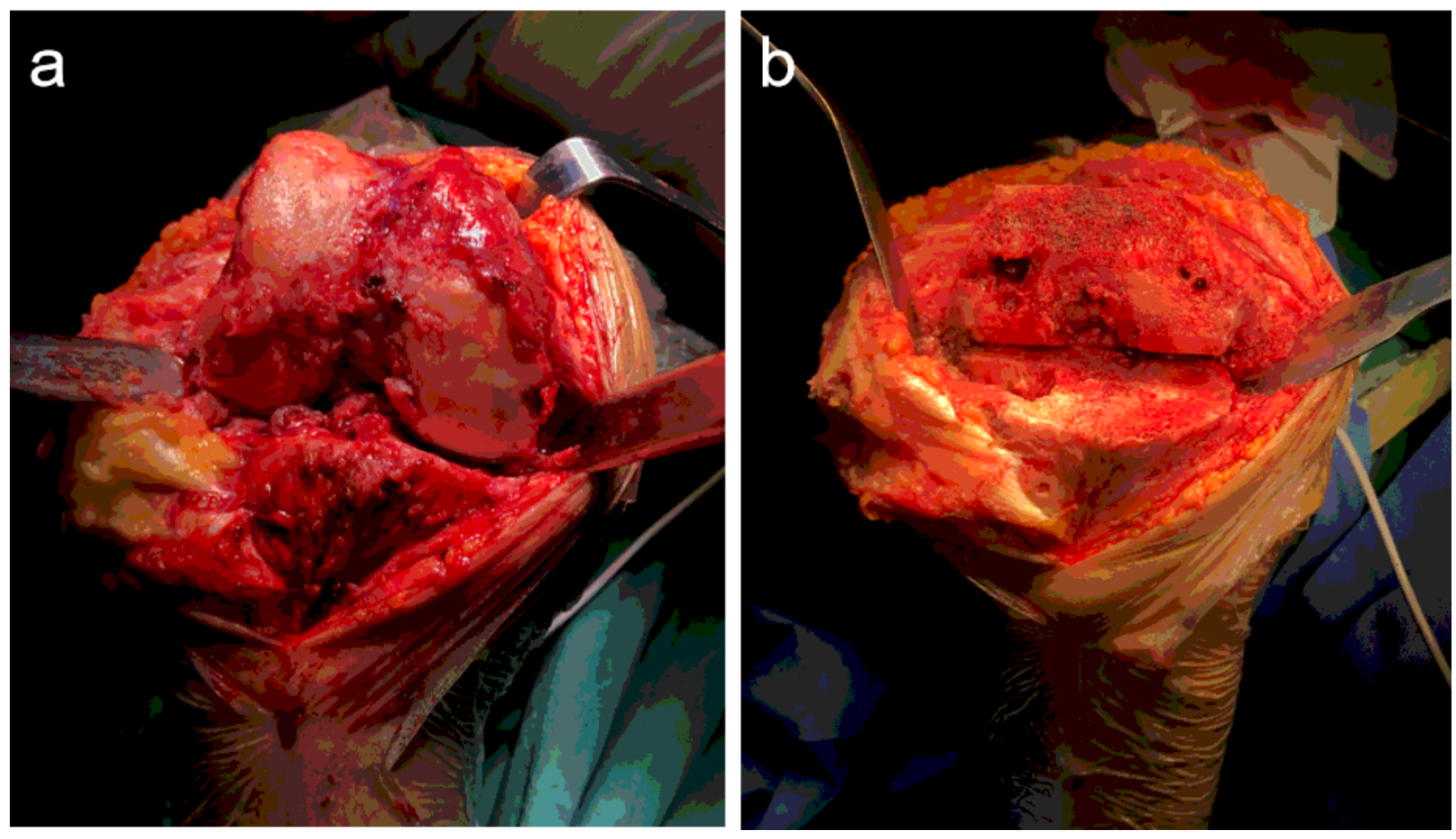

\section{Figure 2}

A: intra-operative images of advanced septic arthritis of knee before debriding; B: image post debridement, extensive synovectomy and bone cutting.
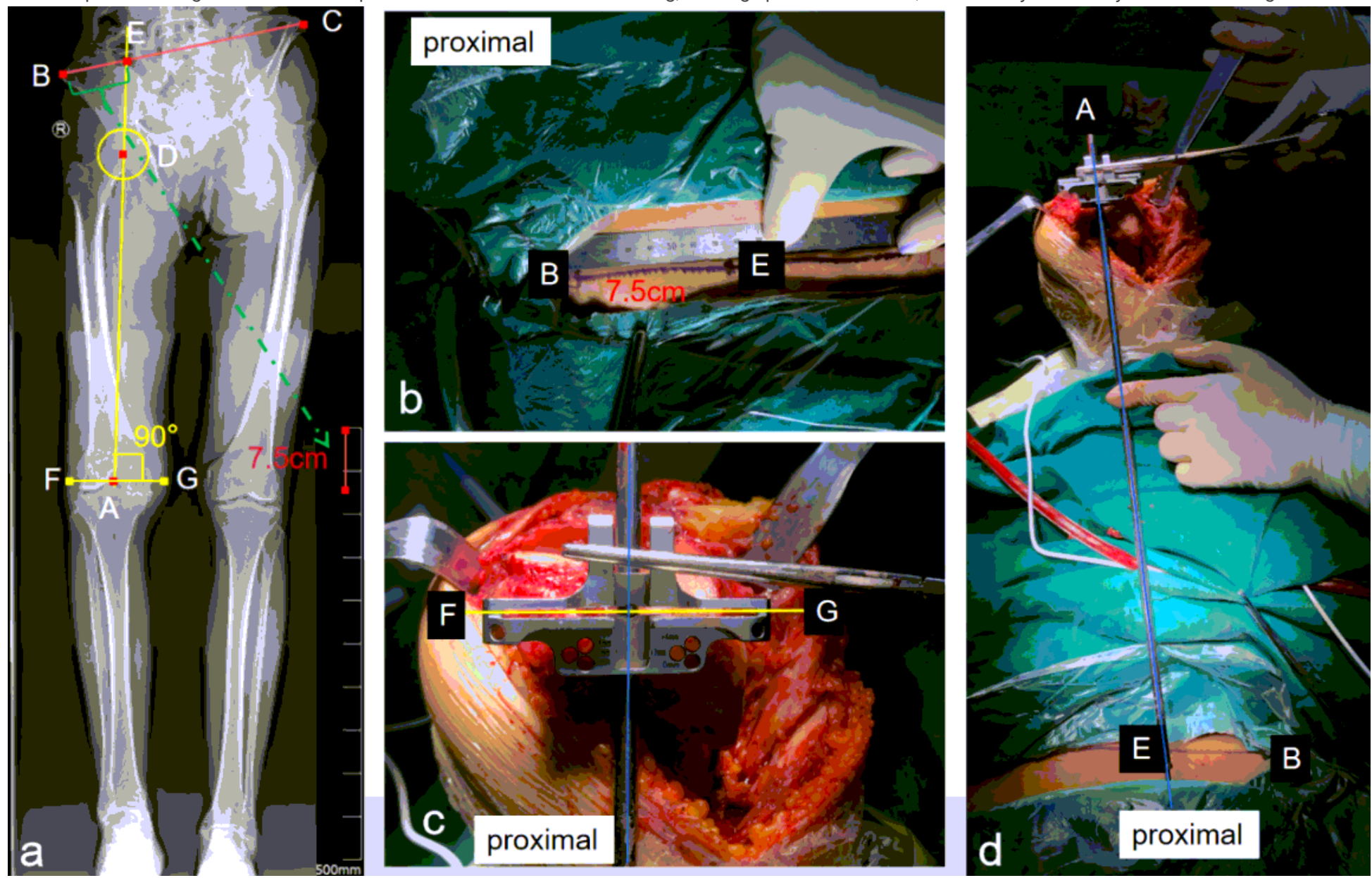

Figure 3

Extramedullary cutting guiding method. (a) Measurements in extramedullary cutting guiding method; (b) Measurement of BE distance on body surface; (c) Final cutting line of distal femur; (d) Mechanical alignment on body surface. 

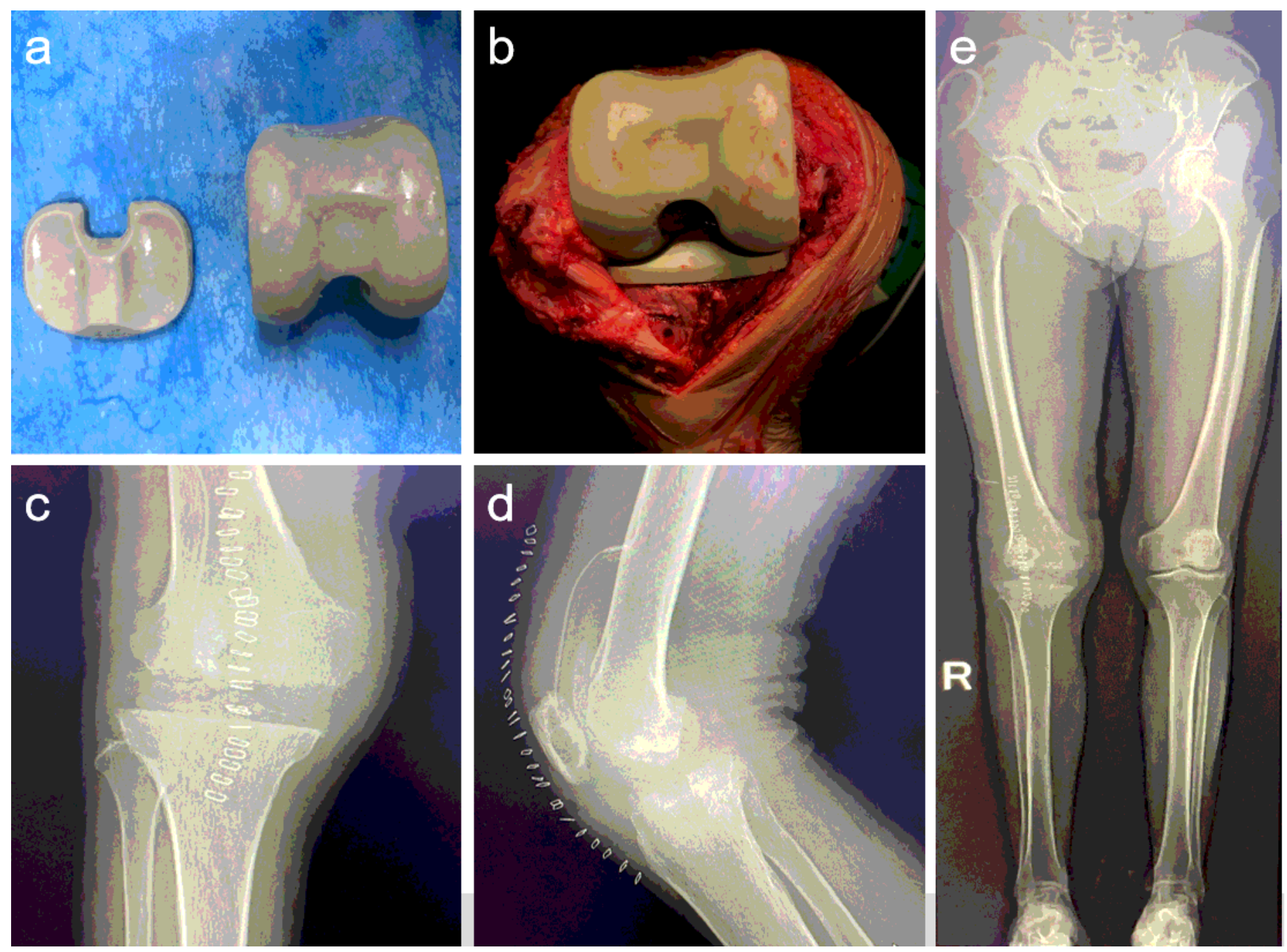

\section{Figure 4}

Images of spacer. (a) Dynamic antibiotic spacer created by a spacer mold; (b) Image of spacer when doing intra-operation; (c) and (d) Immediate postoperative images of AP and lateral views of the spacer; (e) Immediate post-operative full length image of AP view of the spacer. 

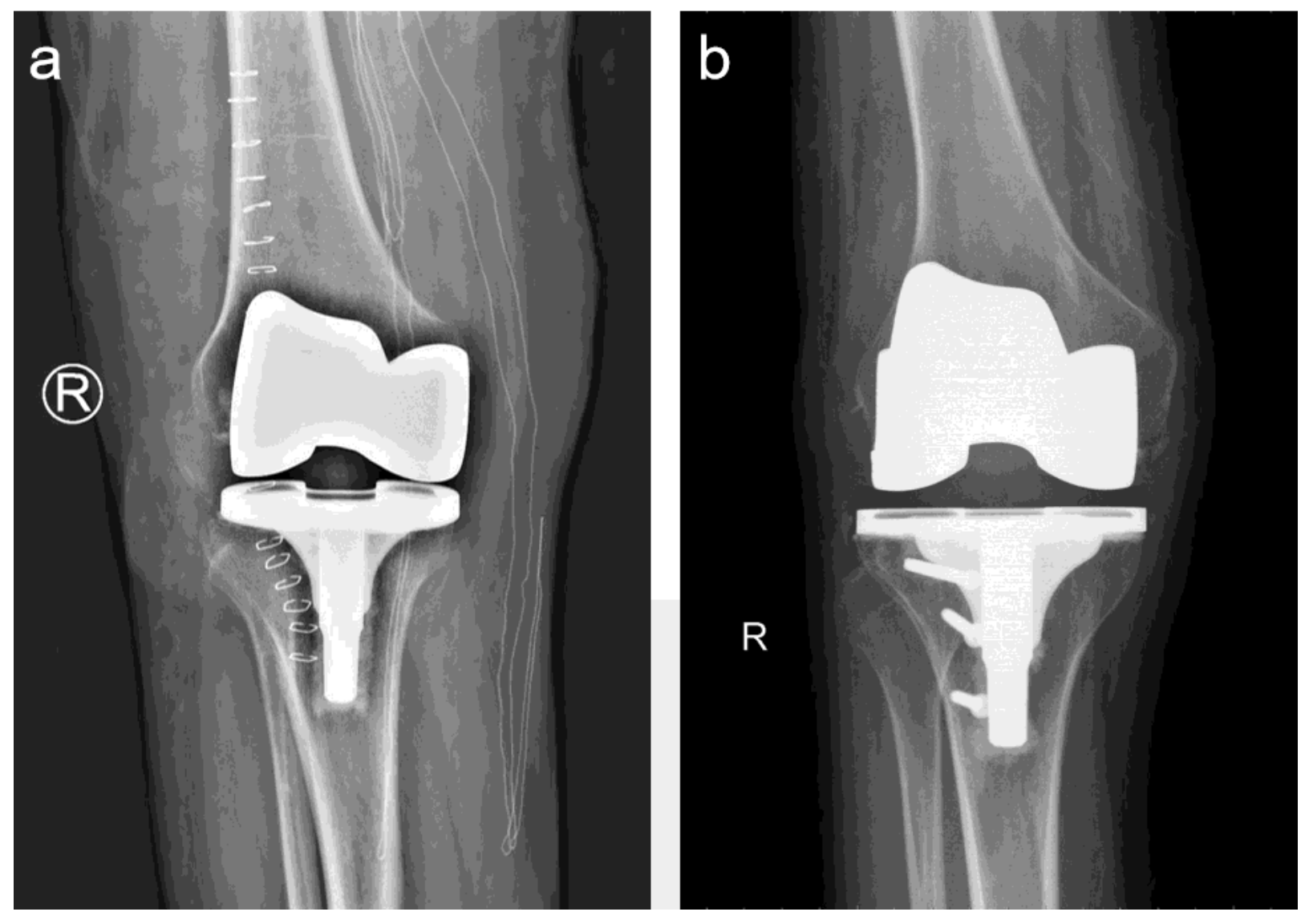

\section{Figure 5}

Knee joint after the second-stage surgery. (a) AP view after second stage operation; (b) AP view after second stage operation with tibial tuberosity osteotomy.
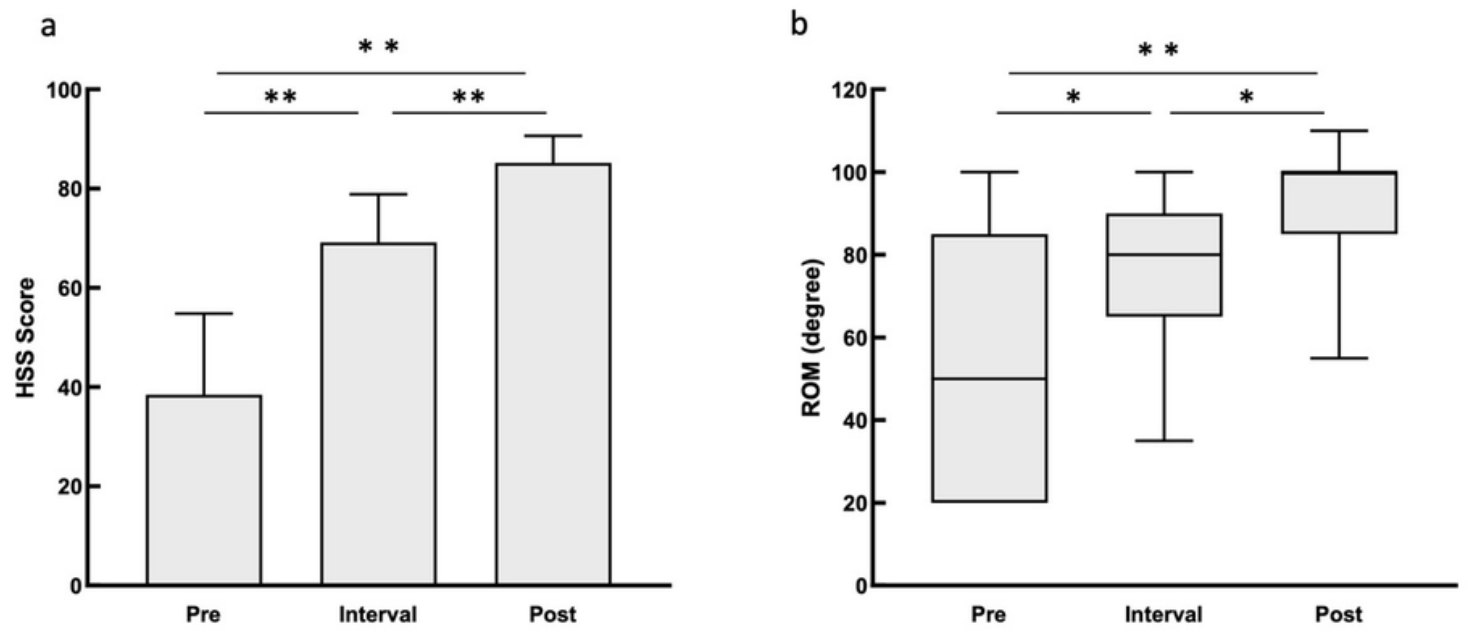

$$
\begin{aligned}
& *<0.05 \\
& * *<0.005
\end{aligned}
$$


Comparison of HSS and ROM. (a) Hospital for Special Surgery Knee score (HSS) among pre-operation, interval period and post-operation; (b) Range of motion (ROM) degree among pre-operation, interval period and post-operation. 